Über die - sehr erheblichen - Wirkungen von kleinen $\mathrm{H}_{2}$-Beimengungen in der positiven Edelgassäule sind z. Zt. Untersuchungen im Gange, über die wir in Kürze berichten werden.

Zum Schluß sei noch kurz eingegangen auf die Frage, inwieweit direkte Absolutbestimmungen von Geschwindigkeitskonstanten bzw. Wirkungsquerschnitten für Ionenreaktionen im Plasma mit der Methode der ambipolaren Effusion möglich erscheinen. Grundsätzlich läßt sich der Betrag des Effusionsstromes einer bestimmten Ionensorte messen und zur Bildungskonstante dieser Ionensorte (etwa $\mathrm{X}^{+}$ nach Reaktion IV) in einfache Beziehung setzen, wenn sicher keine Verluste an der Lochblende auf17 A. V. Phelps u. S. C. Brown, l. c. ${ }^{5}$ und H. J. Oskam, 1. c. ${ }^{7}$. treten. Ferner muß aber noch die Konzentration des geladenen oder des angeregten Reaktionspartners (z. B. bei Reaktion IV die Elektronendichte $\left[\mathrm{e}^{-}\right]$) genügend genau bekannt sein. Dies bedeutet prak tisch noch eine gewisse Schwierigkeit, so daß es vorderhand aussichtsreicher erscheint, Wirkungsquerschnitte in einer Elektronenstoßkammer ${ }^{15}$ oder im abklingenden Plasma zu bestimmen ${ }^{17}$. Die Bedeutung der Effusionsmethode dürfte außer in der Identifizierung der auftretenden Ionen vor allem in der Messung von Verhältnissen der Bildungsgeschwindigkeiten verschiedener Träger unter einigermaßen definierten Plasmazuständen und schließlich noch im Nachweis von neuen Reaktionen zwischen Ionen und neutralen Gasmolekülen liegen.

\title{
Der Einfluß von Sauerstoff, Wasserstoff und Wasserdampf auf die elektrische Leitfähigkeit von Bariumoxyd und Barium:Strontiumoxyd
}

\author{
Von J. Rudolph \\ Aus der OSRAM-Studiengesellschaft, Augsburg \\ (Z. Naturforschg. 13 a, 757-767 [1958]; eingegangen am 17. Mai 1958)
}

\begin{abstract}
Die elektrische Leitfähigkeit $\sigma$ von $\mathrm{BaO}$ bzw. (Ba. Sr) $\mathrm{O}$ in $\mathrm{O}_{2}$, die dem Thermokraftvorzeichen nach eine Defektleitung ist, steigt bei Temperaturen über $\sim 1000^{\circ} \mathrm{K}$ mit dem Sauerstoffdruck $P_{\mathrm{O}_{2}}$ entsprechend $\sigma \sim \mathrm{P}_{2}{ }_{2}^{1 / 0}$ an. Die Gegenwart höher- oder niedrigerwertiger Ionen beeinflußt diese $\mathrm{Ab}$ hängigkeit in charakteristischer Weise: Durch die Gegenwart der einwertigen Alkaliionen wird die p-Leitung in $\mathrm{O}_{2}$ stark erhöht, und $\sigma$ wird über einen größeren $P_{\mathrm{O}_{2}}$-Bereich druckunabhängig. Die höherwertigen Ionen $\mathrm{Y}^{3+}$ oder $\mathrm{La}^{3+}$ erniedrigen $\sigma$ in $\mathrm{O}_{2}$ etwas unter gleichzeitiger Veränderung der Druckabhängigkeit gemäß $\sigma \sim P_{\mathrm{O}_{2}}{ }^{1 / x}$ mit $x<5$. Bei Temperaturen $<1000{ }^{\circ} \mathrm{K}$ treten in $\mathrm{O}_{2}$ und in Gegenwart von Wasserdampfspuren sehr große Leitfähigkeitserhöhungen auf, die offenbar mit der Bildung von Peroxyden im Zusammenhang stehen. $\mathrm{In}_{2}$ liegt beim (Ba, Sr) O Úberschußleitung vor, die sich mit dem Druck gemä $\sigma \sim P_{\mathrm{H}_{2}}{ }^{1 / 4}$ ändert. Auch die Elektronenemission ist unter bestimmten Bedingungen reversibel vom Druck de $\mathrm{H}_{2}$ abhängig. Der Zusammenhang zwischen Leitfähigkeit und Fremdgasdruck sowie der Einfluß von Fremdionen wird diskutiert.
\end{abstract}

Bereits in den dreißiger Jahren wurde in den Arbeiten von Kroczer und LüBke ${ }^{1}$, Meyer und $\mathrm{S}_{\text {ChMidt }}{ }^{2}$ sowie Becker und $\mathrm{SeARS}^{3}$ gezeigt, daß das $\mathrm{BaO}$ bzw. das (Ba, Sr) $\mathrm{O}$ in der in der Oxydkathode vorliegenden aktivierten Form ein Utberschußhalbleiter ist. Andererseits wurde bereits 1939 festgestellt ( Erdalkalioxyde auch in $\mathrm{O}_{2}$ stark ansteigen kann, und $\mathrm{da} ß$ in weniger gut aktivierten Oxydschichten und vor allem in Gegenwart von $\mathrm{O}_{2}$ eine Defektleitung

1 J. Квосzек u. E. Lüвке, Wiss. Veröff. Siemens-Werke 9, 253 [1930].

2 W. Meyer u. A. Schmidt, Z. techn. Phys. 13, 137 [1932].

3 Z. A. Becker u. R. W. Sears, Phys. Rev. 38, 2193 [1931].

4 W. Sсноттку, Z. Elektrochem. 45, 53 [1939] u. briefliche Mitteilung. beobachtbar ist ( $\mathrm{W}_{\text {RIGHT }}{ }^{5}$, Arizumi und Narita ${ }^{6}$ ). Von Ishikawa und Mitarbb. ${ }^{7}$ liegt ein kurzer Bericht über die Änderung der Leitfähigkeit von $\mathrm{BaO}$ in $\mathrm{O}_{2}$ vor. $\mathrm{H}_{\mathrm{AUfF}}$ und $\mathrm{T}_{\mathrm{R}} \ddot{\mathrm{A} N C K L E R}{ }^{8}$ haben an $\mathrm{CaO}$ bei tieferen Temperaturen $\left(600^{\circ}\right)$ eine mit der Chemisorption von $\mathrm{O}_{2}$ in Verbindung stehende Defektleitung festgestellt. Vorstellungen über die Natur der für die p-Leitung verantwortlichen Störzentren beim $\mathrm{BaO}$ sind bisher nicht bekannt.

Auch über die Beschaffenheit der Störstellen in

5 D. A. Wright, Nature, Lond. 164, 714 [1949].

6 T. Arizumi u. S. Narita, Phys. Rev. 85, 388 [1952].

7 Y. Ishikawa, T. Sato, K. Okumura u. T. Sasaki, Phys. Rev. 84, 371 [1951].

8 K. Hauffe u. G. Tränckler, Z. Phys. 136, 166 [1953]. 
einer aktivierten Oxydkathodenschicht, die für die Leitfähigkeit und die Elektronenemission verantwortlich sind, besteht noch keine endgültige Klarheit. Aus dem Verhalten der Leitfähigkeit einer aktivierten Oxydschicht läßt sich kein Schluß auf Störtermlage und Störstellenart ziehen, da die Leitfähigkeit in diesem Fall durch freie Elektronen in den Poren der Schicht (Loosjes und VINK ${ }^{9}$ ) oder im Außenraum um den Meßkörper (Rudolph und Paulisch ${ }^{10}$ ) bestimmt ist. Auf Grund von Untersuchungen anderer, vor allem optischer und lichtelektrischer Art an Einkristallen von $\mathrm{BaO}$ sind eine Reihe von Vorstellungen über die Natur der Donatoren entwickelt worden, die Überschuß-Barium in Form von gelöstem Ba (Redington ${ }^{11}$ ), von F- und $\mathrm{F}^{\prime}$-Zentren (Kane ${ }^{12}$, Sproull, Bever und Libowitz ${ }^{13}$ ), von kolloidalen Ba-Aggregaten (OrTusi ${ }^{14}$ ) u. a. annehmen. In den letzten Jahren zeigte sich indessen, daß offenbar kein quantitativer Zusammenhang zwischen dem Ba-Gehalt und der Emissionsfähigkeit einer Oxydkathode besteht (Moore und Allison ${ }^{15}$ ), und es wurde die

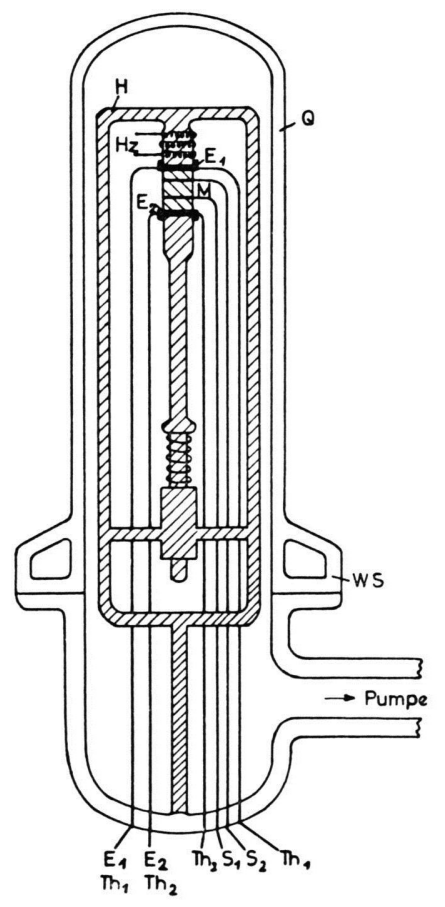

9 R. Loosjes u. H. J. Vink, Philips Res. Rep. 4, 449 u. a. [1949].

10 J. Rudolph u. A. Paulisch, Wiss. Techn. Abh. Osram 7, im Druck.

11 R. W. Redington, Phys. Rev. 87, 1066 [1952].

12 E. O. Kane, J. Appl. Phys. 22, 1214 [1951].

13 R. L. Sproull, R. S. Bever u. G. Libowitz, Phys. Rev. 92, 77 [1953].
Möglichkeit einer Störstellenbildung in der Oxydkathodenschicht unter Mitwirkung der stets - wenn auch in geringer Menge - vorhandenen Restgase diskutiert (Plumlee ${ }^{16}$ ).

Um ganz allgemein Hinweise über die Möglichkeiten der Störstellenbildung und unter Umständen über die Beschaffenheit der Störstellen zu erhalten, wurde die Leitfähigkeit von $\mathrm{BaO}$ und $(\mathrm{Ba}, \mathrm{Sr}) \mathrm{O}$ in Form von Sinterkörpern in verschiedener atmosphärischer Umgebung, wie in $\mathrm{O}_{2}$ und in $\mathrm{H}_{2}$, auch bei gleichzeitiger Anwesenheit von etwas Wasserdampf gemessen. Über den Einfluß dieser Gase auf die Kristall-Leitfähigkeit der Oxyde sowie über die Wirkung verschiedener Fremdionen anderer Wertigkeit wird im folgenden berichtet.

\section{Meßverfahren und Ausgangsstoffe}

Die Leitfähigkeitsmessungen an den in Form von Sinterkörpern vorliegenden Proben erfolgte nach der Sondenmethode, die im einzelnen an anderer Stelle beschrieben wurde ${ }^{17}$. Anordnung und Meßschaltung sind

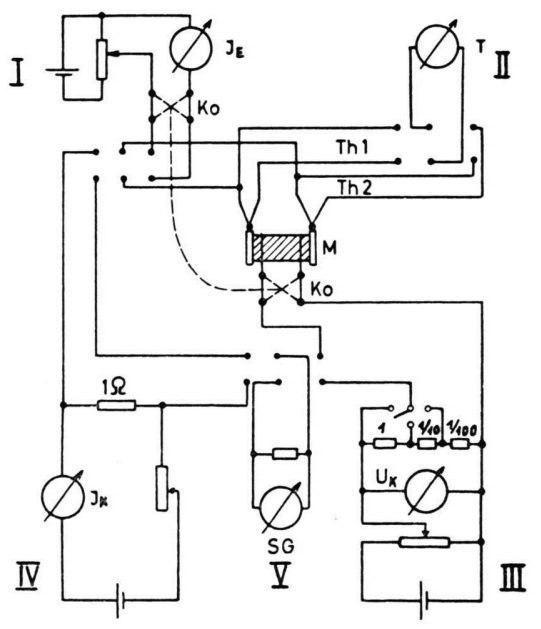

Abb. 1. Meßanordnung und Meßschaltung. Q: Quarzrohr; WS: wassergekühlter Schliff; H: Halter aus Quarz; M: Meßkörper; $\mathrm{E}_{1}, \mathrm{E}_{2}$ : Platinelektroden; $\mathrm{S}_{1}, \mathrm{~S}_{2}$ : Sonden; $\mathrm{Th}_{1}, \mathrm{Th}_{2}$ : Thermoelemente; Hz: Heizung für Thermokraftmessung; I: Einstellung variabler Meßströme; II: Temperaturmessung; III : Sondenspannungskompensation; IV: Thermospannungskompensation; V: Spiegelgalvanometer zur Nullanzeige für III und IV; Ko: Rotierender Kommulator.

14 J. Ortusi, Ann. de Radioelectr. 9, 1 [1954] u. Le Vide 9, 100 [1954].

15 G. E. Moore u. H. W. Allison, J. Appl. Phys. 27, 1396 [1956] und frühere Arbeiten der Verfasser.

16 R. H. Plumlee, RCA-Rev. 17, 190 u. 231 [1956] und J. Appl. Phys. 27, 659 [1956].

17 J. Rudolph, Wiss. Techn. Abh. Osram 7, im Druck. 
schematisch in Abb. 1 wiedergegeben. In dem evakuierbaren Quarzzylinder Q befindet sich zwischen zwei unter Federdruck stehenden Platinelektroden $\mathrm{E}_{1}$ und $\mathrm{E}_{2}$ der Meßkörper $M$, um den die Sonden $S_{1}$ und $S_{2}$ in Form von Platindrähten gelegt sind. Der Strom durch $M$ wird mit dem empfindlichen Instrument $\mathrm{I}_{\mathrm{E}}$ direkt, die Sondenspannung durch Kompensation (Schaltteil III in Schaltskizze) gemessen. Um elektrolytische Effekte auszuschalten, wurde nicht mit Gleichstrom, sondern mit Wechselstrom gearbeitet, der mit Hilfe eines rotierenden Kommutators erzeugt wurde. Dieser Kommutator (Ko in Schaltskizze) polte sowohl den über die Platinelektroden zugeführten Elektrodenstrom sowie die Sondenspannung mit einer zwischen 5 und $100 \mathrm{~Hz}$ wählbaren Frequenz in gleichem Takt um, so daß die Stromund Sondenspanungsmessungen mit Gleichstrominstrumenten ausgeführt werden konnten.

Durch einen über das Quarzgefäß Q geschobenen Heizofen konnte die Temperatur der Meßprobe zwischen Raumtemperatur und etwa $1000{ }^{\circ} \mathrm{C}$ variiert werden. Eine Zusatzheizung $\mathrm{Hz}$ oberhalb der Elektrode $\mathrm{E}_{1}$ erlaubte die Einstellung eines Temperaturgefälles in der Meßprobe und damit die Erzeugung einer Thermospannung, deren Größe durch Kompensation (Schaltteil IV in Meßschaltung) ermittelt werden konnte. Die Temperaturen wurden jeweils mit Hilfe der $\mathrm{Pt}-\mathrm{Pt} / \mathrm{Rh}$-Thermoelemente $\mathrm{Th}_{1}$ und $\mathrm{Th}_{2}$ gemessen.

Mit Hilfe der beschriebenen Anordnung wurde die Leitfähigkeit der Meßproben unter dem Einfluß verschiedener in die Quarzapparatur eingefüllter Gase bei verschiedenen Drucken von $10^{-5}$ bis ca. 500 Torr untersucht. Gleichzeitig konnten das Vorzeichen der Thermokraft und in einzelnen Fällen die Größe der Thermokraft und ihre Temperaturabhängigkeit ermittelt werden.

Als Ausgangsstoffe dienten Substanzen ,zur Analyse“ von Merck, die durch dreimalige Fällung aus Nitratlösungen mit reinsten $\left(\mathrm{NH}_{4}\right)_{2} \mathrm{CO}_{3}$ so weit wie möglich von Verunreinigungen, insbesondere von Alkalien, befreit wurden. Die Analyse ergab nach dieser Reinigung einen $\mathrm{Na}$-Gehalt von weniger als $10^{-5} \mathrm{Mol} \mathrm{Na} / \mathrm{Mol}$ Substanz. Neben diesen reinen Erdalkalikarbonaten wurden auch solche untersucht, die einen Zusatz der niederwertigen Kationen $\mathrm{K}, \mathrm{Na}$ oder $\mathrm{Li}$ bzw. die höherwertigen Kationen $\mathrm{La}^{3^{+}}$oder $\mathrm{Y}^{3^{+}}$enthielten. Die Verunreinigungen durch $\mathrm{K}$ und $\mathrm{Na}$ wurden durch Fällung der Erdalkalikarbonate mit $\mathrm{K}_{2} \mathrm{CO}_{3}$ bzw. $\mathrm{Na}_{2} \mathrm{CO}_{3}$ erhalten. Der Alkaligehalt betrug laut Analyse etwa 0,7 bis 0,8 Gew.-\%. Die höherwertigen Kationen sowie das $\mathrm{Li}$ wurden in bestimmten Mengen als Nitrate vor der Karbonatfällung zugesetzt.

Die als Preßkörper (Zylinderform, Höhe ca. $5 \mathrm{~mm}$, $\varnothing$ ca. $6 \mathrm{~mm}$ ) vorliegenden Karbonate wurden in $\mathrm{CO}_{2}$ vorgesintert und nach Einbau in die Meßapparatur durch Glühen bei ca. $900-1000{ }^{\circ} \mathrm{C}$ unter Pumpen in die Oxyde umgewandelt.

\section{Meßergebnisse}

\section{a) Leitfähigkeit in Sauerstoff}

Die Leitfähigkeit $\sigma$ von $(\mathrm{Ba}, \mathrm{Sr}) \mathrm{O}(1: 1 \mathrm{Mol})$ ist in trockenem Sauerstoff bei Temperaturen über
$1000{ }^{\circ} \mathrm{K}$ gekennzeichnet durch einen nahezu linearen Verlauf von $\log \sigma$ mit der reziproken Temperatur $T^{-1}$ (Abb. 2). Mit zunehmendem $\mathrm{O}_{2}$-Druck erhöht sich die Leitfähigkeit: es zeigt sich eine etwa parallele Verschiebung der Geraden nach höheren Leit-

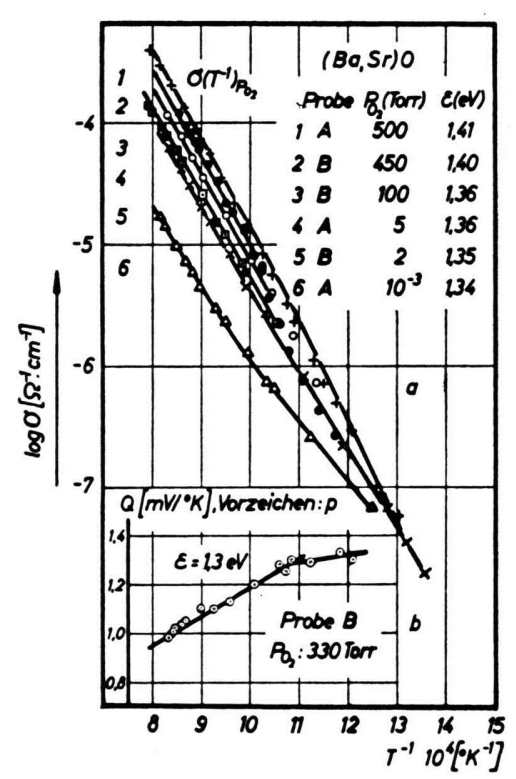

Abb. 2. a) Leitfähigkeit $\sigma$ verschiedener Proben von (Ba, Sr) $\mathrm{O}$ (1:1) als Funktion von $T^{-1}$ bei verschiedenen Sauerstoffdrucken $P_{\mathrm{O}_{2}}$. ( $\varepsilon$ : Aktivierungsenergie.) b) Thermokraft $Q$ als Funktion von $T^{-1}$.

werten. Die Thermokraft hat das Vorzeichen eines p-Leiters, ihr Temperaturverlauf (Abb. 2 b) entspricht bei höheren Temperaturen einer Geraden mit einer ähnlichen Neigung wie bei der Leitfähigkeitsgeraden (Abb. $2 \mathrm{a}$ )* Bei tieferen Temperaturen treten ähnlich wie bei der Leitfähigkeit Abweichungen vom linearen Verlauf auf.

Bei konstanter Temperatur steigt die Leitfähigkeit mit wachsendem Sauerstoffdruck $P_{\mathrm{O}_{2}}$. Die Abhängigkeit der Leitfähigkeit von $P_{\mathrm{O}_{2}}$ (Abb. 3) zeigt im Hochtemperaturgebiet einen Verlauf entsprechend der Beziehung

$$
\sigma \sim P_{\mathrm{O}_{2}}{ }^{1 / x}
$$

mit $x \approx 6$. Noch besser ist diese Änderung der Leitfähigkeit mit der 6. Wurzel aus dem Druck für verschiedene Temperaturen zwischen etwa 1000 und $1250^{\circ} \mathrm{K}$ beim Einfachoxyd $\mathrm{BaO}$ (Abb. 4) erfüllt.

* Die scheinbaren Aktivierungsenergien $\varepsilon$ werden hier offenbar in erster Linie durch die Temperaturabhängigkeit der $\mathrm{O}_{2}$-Aufnahme im Oxyd bestimmt. 


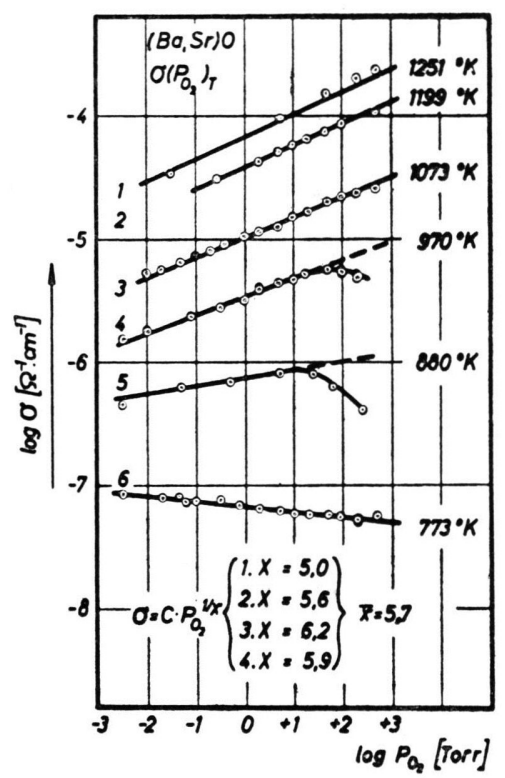

Abb. 3. Leitfähigkeit von (Ba, Sr) O als Funktion des Sauerstoffdruckes $\mathrm{P}_{2}$ bei verschiedenen Temperaturen $T$.

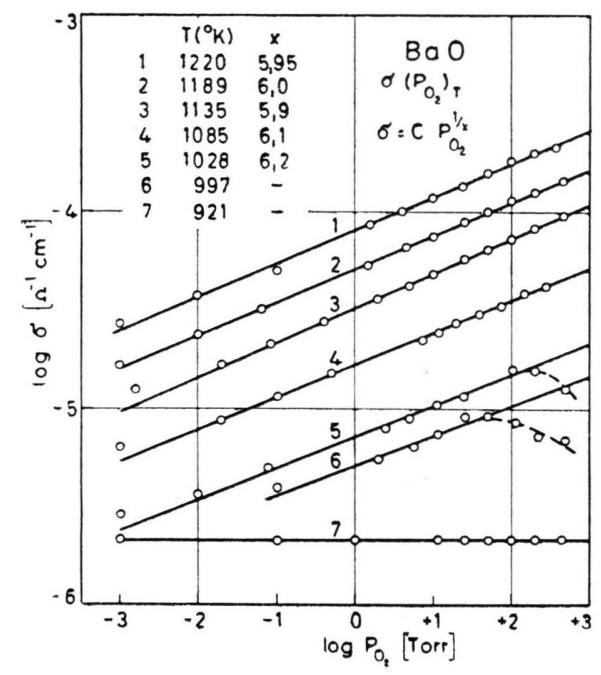

Abb. 4. Leitfähigkeit $\sigma$ von $\mathrm{BaO}$ als Funktion des Sauerstoffdruckes $P_{\mathrm{O}_{2}}$ für verschiedene Temperaturen $T$.

Bei tieferen Temperaturen $\left(<1000{ }^{\circ} \mathrm{K}\right)$ treten im Verlauf von $\log \sigma$ mit $\log P_{\mathrm{O}_{2}}$ stets Abweichungen auf; die Druckabhängigkeit kann ganz verschwinden (Abb. 4, Kurve 7) oder sich sogar mit leichter $\sigma$-Abnahme bei Druckanstieg umkehren (Abb. 3, Kurve 6).

Wird nun der Meßßbereich für die Leitfähigkeit in $\mathrm{O}_{2}$ noch mehr nach tiefen Temperaturen erweitert, so sind im allgemeinen sehr starke Leitfähigkeitsanstiege beobachtbar. Wie Abb. 5 zeigt, ist dann die

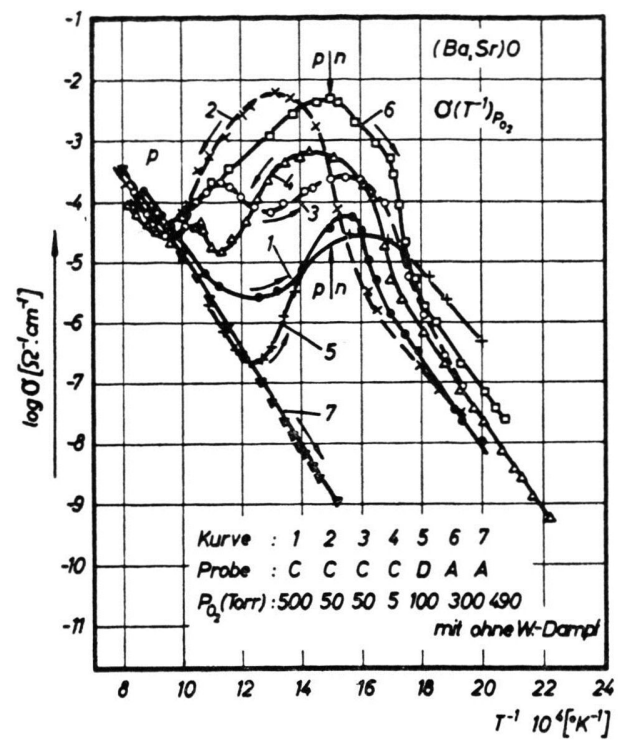

Abb. 5. Leitfähigkeit $\sigma$ von $(\mathrm{Ba}, \mathrm{Sr}) \mathrm{O}$ in $\mathrm{O}_{2}$ als Funktion von $T^{-1}$. Die Pfeile geben die Richtung der Messung in bezug auf Erwärmung oder Abkühlung an.

Leitfähigkeit charakterisiert durch den genannten linearen Bereich bei hohen Temperaturen sowie durch eine lineare Beziehung bei tiefen Temperaturen. Dazwischen liegt ein Úbergangsgebiet mit z. Tl. starkem Anstieg von $\sigma$, wobei der Utbergang, der im besonderen von der Meßgeschwindigkeit und der Richtung im Bezug auf Erwärmung oder Abkühlung abhängig ist, einen hysteresisähnlichen Verlauf besitzt. Häufig ist im Gebiet des Leitfähigkeitsmaximums ein Wechsel des Thermokraftvorzeichens (Abb 5, Kurve 6) beobachtbar.

Die Vermutung, daß der abnorme Leitkurvenverlauf von $(\mathrm{Ba}, \mathrm{Sr}) \mathrm{O}$ mit der Bildung von Peroxyd in Zusammenhang steht, wurde durch Röntgen-Strukturaufnahmen der Massen unmittelbar nach der Messung der charakteristischen Leitkurven bestätigt. Es lag beim ( $\mathrm{Ba}, \mathrm{Sr}) \mathrm{O}$ nach Abkühlung des Meßkörpers in $\mathrm{O}_{2}$ Peroxydstruktur mit gegenüber reinem $\mathrm{BaO}_{2}$ etwas verringerter Gitterkonstante vor ${ }^{* *}$.

Es zeigte sich ferner, daß - in Übereinstimmung mit rein chemischen Untersuchungsergebnissen ${ }^{18}$ eine Peroxydbildung und der damit verbundene Übergang mit erhöhter Leitfähigkeit offenbar nur dann auftritt, wenn gleichzeitig Wasserdampfspuren vorhanden sind; in extrem getrocknetem $\mathrm{O}_{2}$ (Abb. 5, Kurve 7) erfolgt im Gegensatz zu absichtlich mit

\footnotetext{
** Für die Röntgen-Strukturuntersuchungen sei Frau Dr. Schleede-Glassner besonders gedankt.

18 Gmelins Handbuch anorg. Chemie, 8. Aufl., Nr. 30, 94.
} 
etwas Wasserdampf versetztem $\mathrm{O}_{2}$ (Kurve 6) ein nahezu linearer $\sigma$-Verlauf bis zu tiefen Temperaturen.

Auch der Temperaturbereich der Peroxydbildung und der Peroxydbeständigkeit nach den chemischen Befunden ${ }^{19}$ kommt im Bild der Leitkurven zum Ausdruck. Beim Erhitzen von - zuvor im Vakuum geglühtem - $(\mathrm{Ba}, \mathrm{Sr}) \mathrm{O}$ in $\mathrm{O}_{2}$ (mit etwas Wasserdampf) tritt etwa bei gleichen Temperaturen Bildung von Peroxyd (Abb. 6b) und $\sigma$-Anstieg (Abb. 6a) ein; im Gebiet der 100-proz. Beständigkeit des Peroxyds fällt $\sigma$ (Stöchiometrie) stark ab, im Gebiet der Zersetzung bei höheren Temperaturen steigt $\sigma$ wieder sprunghaft an (Unstöchiometrie) und erreicht nach vollständiger Zersetzung in das Oxyd wieder den linearen $\sigma\left(T^{-1}\right)$-Verlauf.

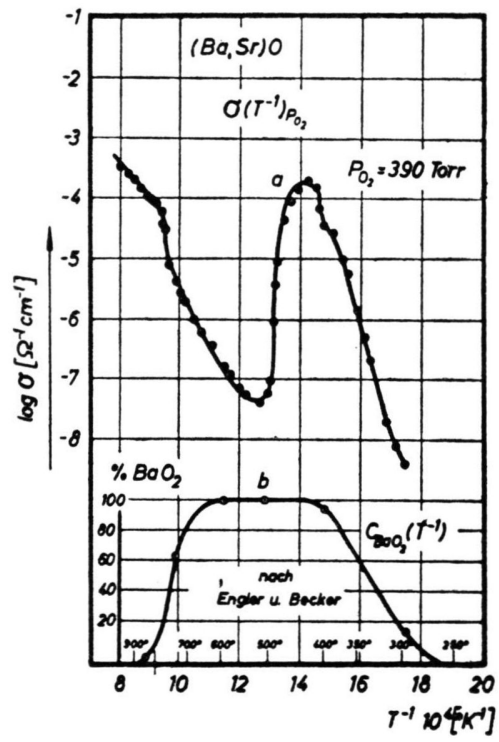

Abb. 6. a) Leitfähigkeit $\sigma$ von ( $\mathrm{Ba}, \mathrm{Sr}) \mathrm{O}$ beim Aufheizen in feuchtem $\mathrm{O}_{2}$. b) Menge des sich bildenden $\mathrm{BaO}_{2}$ beim Aufheizen von $\mathrm{BaO}$ in feuchtem $\mathrm{O}_{2}$ nach ENGLER und BeCKer ${ }^{19}$.

Durch Fremdstoffzusätze zum (Ba, Sr) O wird dessen Leitfähigkeit charakteristisch verändert. Geringe Mengen von Alkaliionen erhöhen die Defektleitung beträchtlich und - wie Abb. 7 zeigt - in steigendem Maße in der Reihenfolge $\mathrm{Li}-\mathrm{Na}-\mathrm{K}$. Jedoch geht beim $\mathrm{Na}$ - und K-haltigen Erdalkalioxyd die Leitfähigkeitserhöhung während der Messungen als Folge einer Verdampfung des Alkalis allmählich zu-

19 C. Engler u. W. Becker, ebd. S. 95 u. Ber. Heidelberg. Akad. 1, 6 [1909/10].

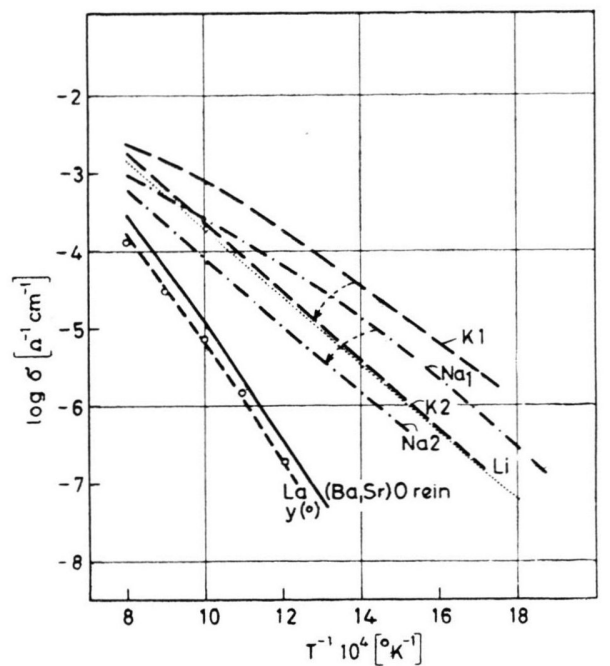

Abb. 7. Leitfähigkeit $\sigma$ in $\mathrm{O}_{2}$ als Funktion von $T^{-1}$ für $(\mathrm{Ba}, \mathrm{Sr}) \mathrm{O}$ mit verschiedenen Fremdstoffzusätzen. (Ba, Sr) O - rein: Ohne Zusätze, $P_{\mathrm{O}_{2}}: 400$ Torr. La(Y) : Mit 0,5 Mol- ${ }^{\circ}$ La (bzw. Y), $\mathrm{P}_{2}$ : 380 Torr. $\mathrm{Kl}$ bzw. K2: $\mathrm{Mit} \mathrm{K}_{2} \mathrm{CO}_{3}$ gefällt, $P_{\mathrm{O}_{2}}$ : 173 (bzw. 380) Torr; K1 zu Beginn, K2 gegen Ende der Messungen. Nal bzw. Na2: Mit $\mathrm{Na}_{2} \mathrm{CO}_{3}$ gefällt, $\mathrm{P}_{\mathrm{O}_{2}}$ : 218 (bzw. 380) Torr; Nal zu Beginn, Na2 zum Ende der Messungen. $\mathrm{Li}$ : Mit 0,5 Mol-\% Li, $\mathrm{P}_{2}: 380$ Torr.

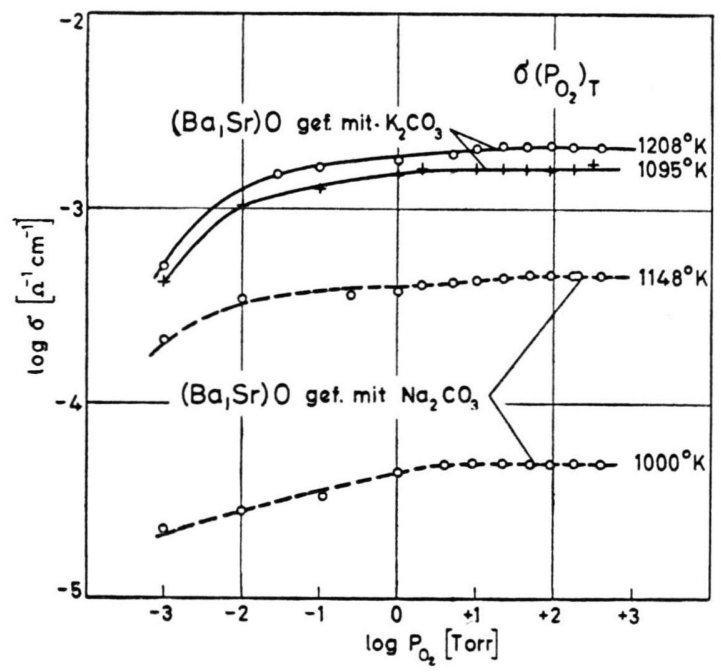

Abb. 8. Leitfähigkeit $\sigma$ von $\mathrm{Na}_{2} \mathrm{CO}_{3}$ - bzw. $\mathrm{K}_{2} \mathrm{CO}_{3}$-gefälltem (Ba, Sr) $\mathrm{O}$ als Funktion des Sauerstoffdruckes $\mathrm{P}_{\mathrm{O}_{2}}$.

rück (vgl. die ersten und zweiten Leitfähigkeitsmessungen an Hand der Kurven $\mathrm{Kl}$ und K2 bzw. Nal und Na2). Auf Grund einer Analyse war im Verlauf einiger Messungen im Vakuum und in $\mathrm{O}_{2}$ der ursprüngliche K-Gehalt einer Probe von $0,77 \%$ auf $2,5 \cdot 10^{-3} \%$ zurückgegangen. Das Thermokraftvorzeichen entspricht wieder dem eines Defektleiters. 


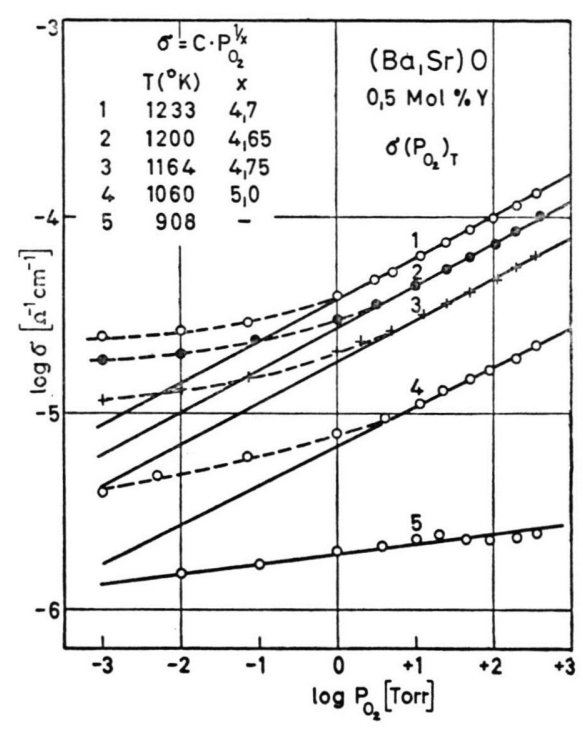

Abb. 9. Leitfähigkeit $\sigma$ von ( $\mathrm{Ba}, \mathrm{Sr}) \mathrm{O}$ mit $0,5 \mathrm{Mol}-\% \mathrm{Y}$ als Funktion des Sauerstoffdruckes $\mathrm{P}_{2}$.

Gleichzeitig zeigt Abb. 7, daß die Gegenwart der höherwertigen Ionen La und Y die Leitfähigkeit des (Ba, Sr) $\mathrm{O}$ in $\mathrm{O}_{2}$ ein wenig (etwa um den Faktor 2) verringert.

Der Einfluß der Fremdionen ist im besonderen in der Form der $\mathrm{O}_{2}$-Druckabhängigkeit der Leitfähigkeit zu beobachten. Bei den alkalihaltigen Oxyden ist die Leitfähigkeit über einen größeren $\mathrm{O}_{2}$-Druckbereich nahezu druckunabhängig und erst bei kleinen Drucken tritt eine zeitlich nur langsam erfolgende Leitfähigkeitsabnahme ein (vgl. Abb. 8). Dagegen sinkt bei den Oxyden mit den höherwertigen Kationen $\mathrm{Y}^{3^{+}}$und $\mathrm{La}^{3^{+}}$die Leitfähigkeit bereits von höchsten $\mathrm{O}_{2}$-Drucken mit fallendem $P_{\mathrm{O}_{2}}$ rasch ab (vgl. Abb. 9 und 10). Aber bereits bei mittleren Drucken von 1 bis 10 Torr beginnt $\sigma$ vom linearen Verlauf abzuweichen und steigt mitunter mit abnehmendem $P_{\mathrm{O}_{2}}$ sogar wieder an (Abb. 10, Kurve 1). Im Gebiet des Minimums wird die Thermokraft, die im Gebiet höheren Druckes eindeutig das Vorzeichen eines p-Leiters hat, unmeßbar gering, so daß eine Vorzeichenbestimmung nicht mehr gelingt. Es erfolgt offenbar in diesem Druckbereich bereits ein Übergang von $\mathrm{p}$ - zu n-Leitung.

Im Gebiet der $\sigma$-Abnahme mit sinkendem $P_{\mathrm{O}_{2}}$ ergibt sich für die Oxyde mit höherwertigen Fremdionen ebenfalls eine lineare Beziehung

$$
\sigma \sim P_{02}{ }^{1 / x},
$$

wobei $x$ im allgemeinen kleiner als 6 ist und darüber

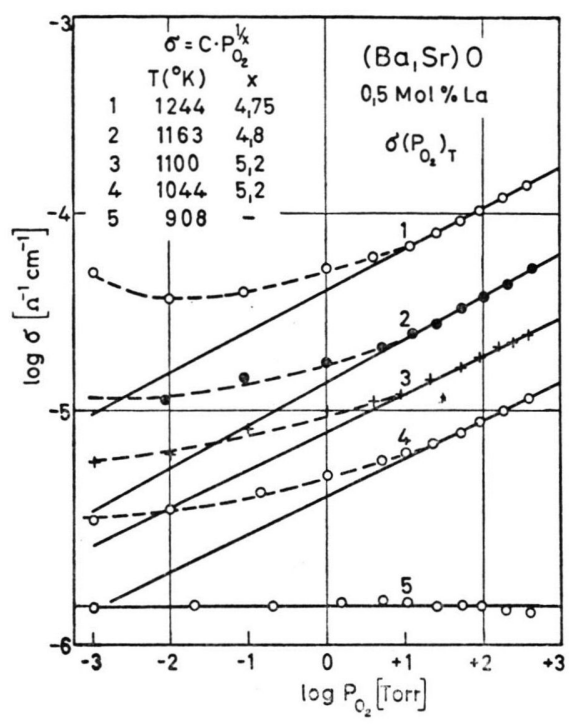

Abb. 10. Leitfähigkeit $\sigma$ von (Ba, Sr) $\mathrm{O}$ mit $0,5 \mathrm{Mol} \% \mathrm{La}$ als Funktion des Sauerstoffdruckes $\mathrm{P}_{2}$.

hinaus mit steigender Temperatur offenbar die Tendenz einer Abnahme zeigt (Abb. 9 und 10).

\section{b) Leitfähigkeit in Wasserstoff}

Ein im Charakter zunächst ähnliches Bild wie in $\mathrm{O}_{2}$ ergibt sich für die in Wasserstoff gemessene Leitfähigkeit von $(\mathrm{Ba}, \mathrm{Sr}) \mathrm{O}$. Auch hier erfolgt zwischen einem linearen Bereich von $\log \sigma$ über $T^{-1}$ bei hohen $\left(>1000^{\circ}\right)$ und tiefen Temperaturen ein Übergang mit starkem Leitfähigkeitsanstieg. Die Pfeile an den Kurven (Abb. 11) geben jeweils die Richtung der Messung in bezug auf Erwärmung bzw. Abkühlung an. Das Thermokraftvorzeichen entspricht bei hohen Temperaturen dem eines Überschußleiters.

Die Abhängigkeit der Leitfähigkeit vom $\mathrm{H}_{2}$-Druck $P_{\mathrm{H}_{2}}$ ist in den verschiedenen Temperaturgebieten unterschiedlich. Unter der Voraussetzung, daß das ( $\mathrm{Ba}, \mathrm{Sr}) \mathrm{O}$ vor dem Einlassen von $\mathrm{H}_{2}$ in nicht-aktiviertem Zustand vorliegt (Kurve 4), erfolgt bei hohen Temperaturen $\left(>1000^{\circ} \mathrm{K}\right)$ beim Einlassen oder Abpumpen des $\mathrm{H}_{2}$ ein reversibler Übergang zwischen den Leitkurven mit höchsten und niedrigsten Leitwerten (siehe Pfeil a und b in Abb. 11). Im Mitteltemperaturgebiet wächst bei Einlassen von $\mathrm{H}_{2}$ die Leitfähigkeit von Werten der Vakuumkurve (Kurve 4) über etwa 7 Zehnerpotenzen bis zu Werten der $\mathrm{H}_{2}$ Kurve 2 (Pfeil c); beim Abpumpen des $\mathrm{H}_{2}$ jedoch zeigt sich eine Abnahme von $\sigma$ nur mehr bis auf einen etwa auf der Verlängerung der Tieftemperatur- 
geraden liegenden Wert (Pfeil d bzw. e). Die zeitliche Änderung von $\sigma$ beim Einlassen und Abpumpen von $\mathrm{H}_{2}$ in den verschiedenen Temperaturbereichen zeigt Abb. 12.

Es wird vermutet, daß das Verhalten der Leitfähigkeit bei tiefen Temperaturen auch von - wahrscheinlich sich stets bildenden - geringen Wasserdampfspuren beeinflußt wird. Eine eindeutige $\mathrm{Zu}$ ordnung ist bisher nicht möglich.
Bei Temperaturen über etwa $1000{ }^{\circ} \mathrm{K}$ wird eine Zunahme der Überschußleitfähigkeit mit dem Wasserstoffdruck beobachtet, die quantitativ die Beziehung

$$
\sigma \sim P_{\mathrm{H} 2}{ }^{1 / 4}
$$

befolgt. Abb. 13 zeigt einige dieser Druckabhängigkeiten bei verschiedenen Temperaturen. Bei tiefen Temperaturen gilt diese Beziehung nicht mehr (Kurve 5, Abb. 13).

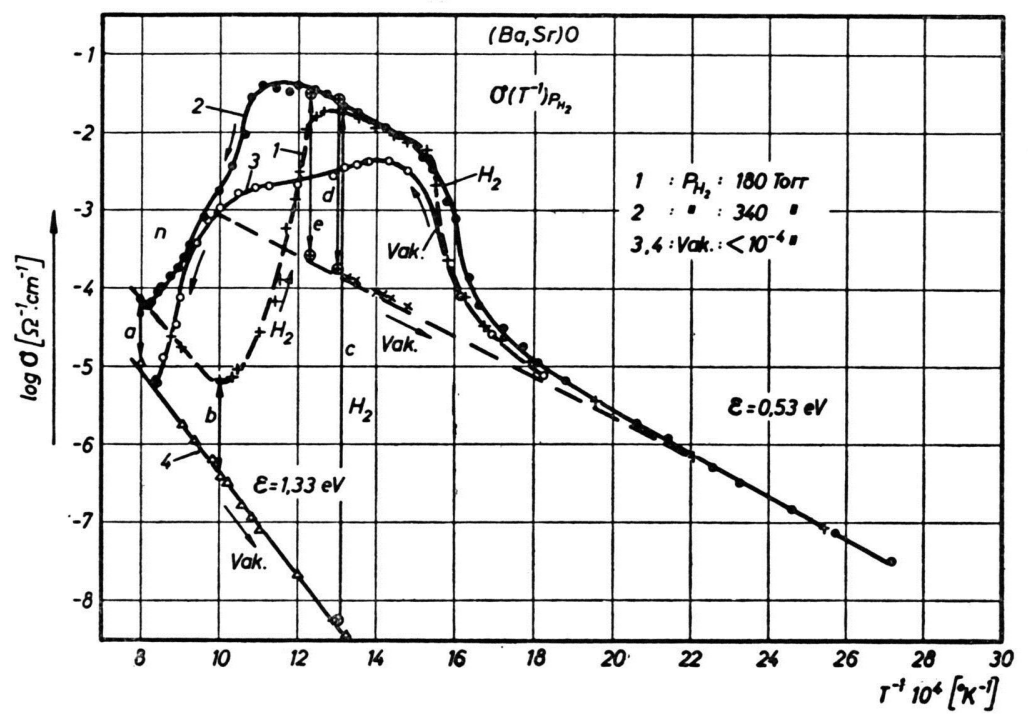

Abb. 11. Leitfähigkeit $\sigma$ von $(\mathrm{Ba}, \mathrm{Sr})$ in $\mathrm{H}_{2}$ als Funktion von $T^{-1}$. Die Pfeile a bis e geben die Änderung von $\sigma$ beim Einlassen bzw. Abpumpen des $\mathrm{H}_{2}$ an.

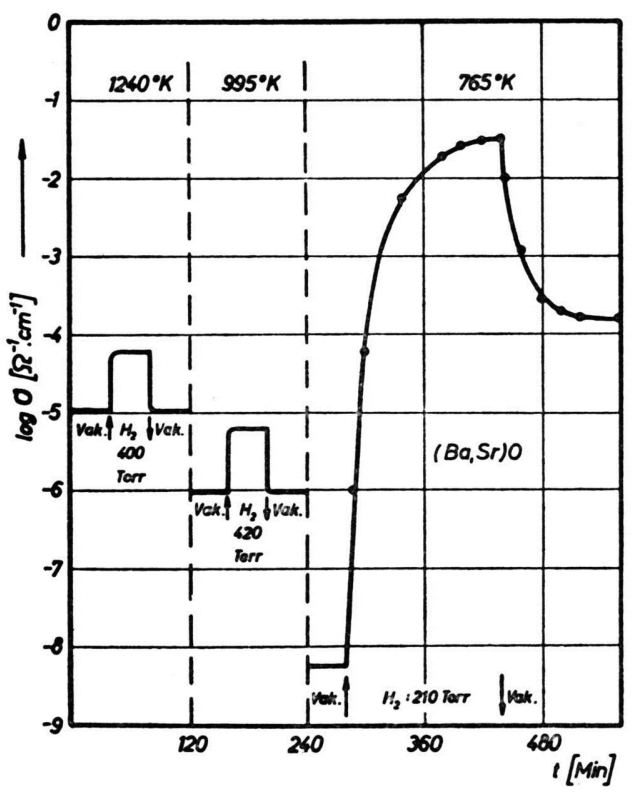

Abb. 12. Zeitliche Änderung der Leitfähigkeit $\sigma$ von (Ba, Sr) O beim Einlassen bzw. Abpumpen von $\mathrm{H}_{2}$.

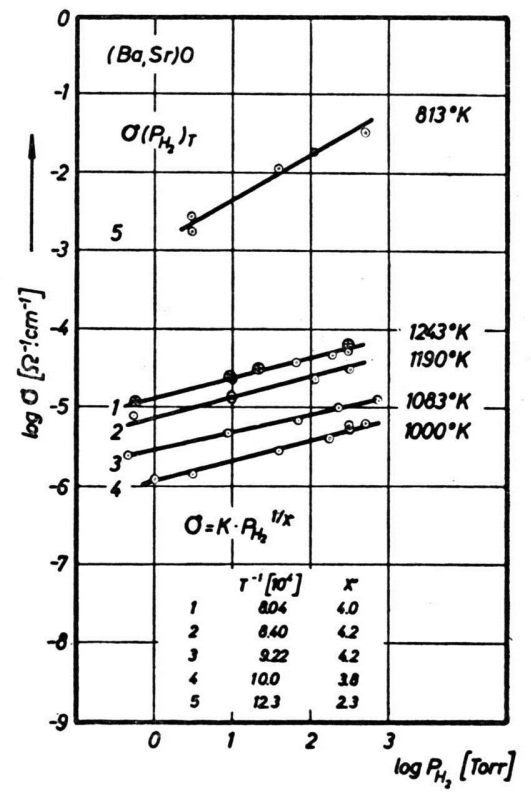

Abb. 13. Die Änderung der Überschußleitung v. (Ba, Sr) $\mathrm{O}$ mit dem Druck $P_{\mathrm{H}_{2}}$ des $\mathrm{H}_{2}$ bei verschiedenen Temperaturen $T$. 
Es liegt also auch in $\mathrm{H}_{2}$ - ähnlich wie im $\mathrm{O}_{2}$, jedoch mit umgekehrten Trägervorzeichen - eine Leitfähigkeitszunahme mit dem Druck im Sinne einer Beteiligung von $\mathrm{H}_{2}$ am Leitfähigkeitsmechanismus vor. Sehr wahrscheinlich handelt es sich hierbei um eine durch die Wasserstoffbeteiligung modifizierte Kristalleitfähigkeit und nicht um eine Leitung durch freie Elektronen der erwähnten Art (l. c. ${ }^{9,10}$ ), da die Leitfähigkeit über freie Elektronen in $\mathrm{H}_{2}$ - wie im folgenden kurz gezeigt wird - einen anderen Verlauf der Abhängigkeit vom Druck besitzt.

\section{c) Elektronenemission in Wasserstoff}

Die Frage, wieweit die Gegenwart von Wasserstoff außer auf die Leitfähigkeit auch auf die Elektronenemission von $(\mathrm{Ba}, \mathrm{Sr}) \mathrm{O}$ von $\mathrm{Einfluß}$ ist, wurde an Hand einiger Emissionsmessungen kurz untersucht.

So wurde zunächst in der gleichen Meßanordnung, die nur zusätzlich eine Anode in Form eines PtDrahtrings um den zylindrischen Meßkörper im Abstand von etwa $5 \mathrm{~mm}$ enthielt, an einer im Vakuum befindlichen, unaktivierten $(\mathrm{Ba}, \mathrm{Sr})$ O-Probe eine beim Einlassen von etwa 1 Torr $\mathrm{H}_{2}$ auftretende Elektronenemission (Anodenspannung $6 \mathrm{~V}$ ) beobachtet, die bei schnellem Abpumpen des $\mathrm{H}_{2}$ rasch wieder verschwand. Zeitlicher Anstieg des Elektronenemissionsstromes $J_{\mathrm{e}}$ bei $\mathrm{H}_{2}$-Einlaß und zeitlicher Abfall beim Abpumpen wurden mit einem Schleifenoszillographen aufgenommen. Der Verlauf ist in Abb. 14 wiedergegeben.

Ferner wurde ebenfalls an einer unaktivierten (Ba, Sr) O-Probe der Emissionsstrom bei konstanter Temperatur als Funktion des $\mathrm{H}_{\mathbf{2}}$-Druckes gemessen (Abb. 15). Es zeigt sich, daß $J_{\mathrm{e}}$ zunächst mit $P_{\mathrm{H} 2}$ bis zu einem Maximum zwischen 0,1 und 1 Torr ansteigt, dann aber mit $P_{\mathrm{H} 2}$ wieder absinkt (ausgezogene Kurve 1). Die Abnahme von $J_{e}$ bei größeren $\mathrm{H}_{2}$-Drucken dürfte sich dadurch erklären, daß die freie Weglänge der Elektronen zwischen Kathode (Oxyd) und Anode mit $P_{\mathrm{H} 2}$ von einem bestimmten Wert an, nämlich wenn $P_{\mathrm{H}_{2}}$ groß genug ist, um den freien Flug der Elektronen zwischen Kathode und Anode zu behindern, abnehmen muß. Theoretisch sollte $J_{\mathrm{e}}$ dann mit $P_{\mathrm{H} 2}{ }^{-1}$ abnehmen (gestrichelte Kurve 2). Die gemessene Abnahme erfolgt indessen nur mit $P_{\mathrm{H} 2}{ }^{-1 / 2,5}$, so daß sich aus der Differenz beider Kurven - wie in Abb. 15, Kurve 3, graphisch gezeigt ist - eine Zunahme des Emissionsstromes $J_{\mathrm{e}}$

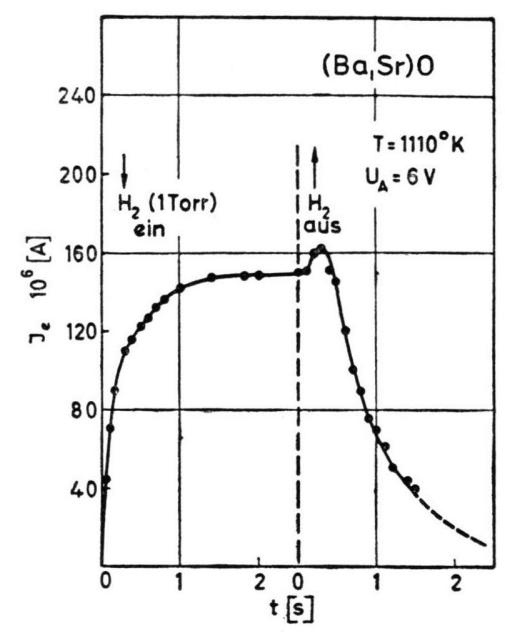

Abb. 14. Die zeitliche Änderung des Emissionsstromes $J_{e}$ von (Ba, Sr) $\mathrm{O}$ beim Einlassen bzw. Abpumpen von $\mathrm{H}_{2}$ (1 Torr).

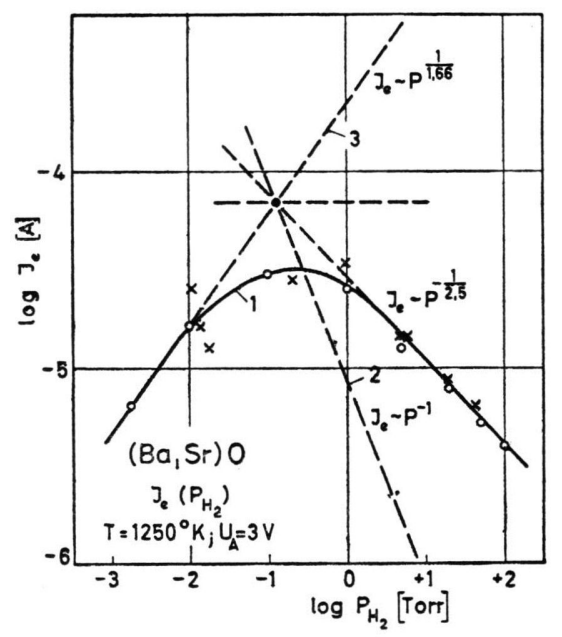

Abb. 15. Die Änderung des Emissionsstromes $J_{\mathrm{e}}$ als Funktion des $\mathrm{H}_{2}$-Druckes.

entsprechend der Beziehung

$$
J_{\mathrm{e}} \sim P_{\mathrm{H} 2}{ }^{1 / 1,66}
$$

ergibt.

Es zeigt sich also, daß offenbar der Wasserstoff auch Störzentren, die zu einer Elektronenemission führen, im $(\mathrm{Ba}, \mathrm{Sr}) \mathrm{O}$ zu bilden vermag. Es sei wie schon oben - an dieser Stelle vermerkt, daß der Druckabhängigkeitsverlauf von $J_{\mathrm{e}}$ ganz anders als der lineare Verlauf der Leitfähigkeit mit $P_{\mathrm{H}_{2}}$ (Abb. 13 ) ist. Es liegt also offenbar bei der Leitfähigkeitsmessung tatsächlich eine Leitung über Elektronen im Kristall und nicht über freie emittierte Elektronen wie beim Leitungsmechanismus eines gut aktivierten Oxyds vor. 


\section{Diskussion}

Als wesentliche Merkmale ergeben sich aus den geschilderten Messungen:

1. Die p-Leitung des Einfach- bzw. Doppeloxyds ändert sich bei höheren Temperaturen in Sauerstoff proportional zur 6. Wurzel aus dem Druck $P_{\mathrm{O}_{2}}$.

2. Es zeigt sich beim Doppeloxyd in Gegenwart höherwertiger Fremdionen eine Abnahme des Wurzelexponenten (bis etwa 4,7).

3. Durch Alkaliionen wird die Druckabhängigkeit der p-Leitung im Bereich höherer $\mathrm{O}_{2}$-Drucke praktisch aufgehoben.

4. In $\mathrm{H}_{2}$ liegt für $T>1000{ }^{\circ} \mathrm{K}$ eine n-Leitung vor, die sich proportional mit der 4 . Wurzel aus dem Wasserstoffdruck ändert.

Der empirischen Abhängigkeit $\sigma \sim P_{\mathrm{O} 2}{ }^{1 / x}$ mit $x=6$ würde die Vorstellung entsprechen, da $\beta$ bei hohen Temperaturen der Sauerstoff $\mathrm{O}_{2 \mathrm{~g}}$ im Kristall gelöst wird $\left(\mathrm{O}_{\mathrm{f}}\right)$ und positive Löcher zur Leitung beisteuert entsprechend:

$$
\mathrm{O}_{2 \mathrm{~g}} \rightleftarrows 2 \mathrm{O}_{\mathrm{f}}^{2^{-}}+4 \oplus \text {. }
$$

Nach dem Massenwirkungsgesetz ergibt sich

$$
K P_{0_{2}}=\left[\mathrm{O}_{\mathrm{f}}{ }^{2-}\right]^{2} \cdot[\oplus]^{4} \text {. }
$$

Da die Konzentration des gelösten $\mathrm{O}_{\mathrm{f}}{ }^{2-}$ der Konzentration der Löcher proportional ist und diese wiederum der Leitfähigkeit proportional ist, wird

$$
\sigma=K^{\prime} P_{02}{ }^{1 / 6}
$$

entsprechend dem für $\mathrm{BaO}$ geltenden Befund. Danach wäre eine von Temperatur und $\mathrm{O}_{2}$-Druck abhängige Aufnahme von $\mathrm{O}_{2}$ im Kristall anzunehmen.

Grundsätzlich sind bezüglich der Beeinflussung der Leitfähigkeit eines Festkörpers durch Gase auch Adsorptions- und Chemisorptionsmechanismen mit veränderter Störstellenbildung an Oberflächen oder in Oberflächenschichten insbesondere bei polykristallinen Körpern zu berücksichtigen. Die Annahme reiner Adsorptionseffekte, die bei den relativ hohen Temperaturen nicht wahrscheinlich sind, würde eine Druckabhängigkeit der Leitfähigkeit im Sinne einer LANGMUIrschen Adsorptionsisothermen verlangen und widerspricht aus diesem Grunde dem beobachteten Verhalten. Das Vorliegen einer Chemisorption von $\mathrm{O}_{2}$ mit bevorzugter Defektleitung in Oberflächenzonen der Sinterkörper ließe sich eindeutig nur durch Messungen an BaO-Einkristallen ausschalten. Indessen sprechen eine Reihe von Gesichtspunkten mehr für eine homogene Auflösung des $\mathrm{O}_{2}$ im Kristall als für eine Chemisorption, wie sie etwa von $\mathrm{H}_{\text {Auffe }}{ }^{8}$ für das Verhalten von $\mathrm{CaO}$ bei tiefen Temperaturen $\left(600^{\circ} \mathrm{C}\right)$ angenommen wird; in diesem Tieftemperaturbereich $\left(<730{ }^{\circ} \mathrm{C}\right)$ gilt ja auch für $\mathrm{BaO}$ nach unseren Beobachtungen das $P_{02}{ }^{1 / 6}$. Gesetz nicht mehr.

Wie Timmer ${ }^{20}$ zeigte, löst sich bei hohen Temperaturen in $\mathrm{BaO}$-Einkristallen, die sich im Ba-Dampf verschiedenen Druckes $P_{\mathrm{Ba}}$ befinden, das $\mathrm{Ba}$ unter Bildung homogen verteilter Farbzentren auf, wobei die Farbzentrenkonzentration - im Einklang mit einem entsprechenden Ansatz des Massenwirkungsgesetzes - proportional zu $P_{\mathrm{Ba}}{ }^{1 / 3}$ ist. Die Bildung dieser Farbzentren kann als ein Ausbau von $\mathrm{O}$ aus dem Kristall unter Neuaufbau von $\mathrm{BaO}$ an der Kristalloberfläche angesehen werden. Bei hohen Tem. peraturen sollte daher eine leichte Diffusion von Sauerstoff (dessen Ionenradius im übrigen kleiner als der der $\mathrm{Ba}^{2+}$-Ionen ist) im Kristall möglich sein. In der Tat wurde von Sproull und Mitarb. ${ }^{13}$ in ihren Untersuchungen über die additive Verfärbung von $\mathrm{BaO}$-Kristallen nachgewiesen, daß bei Temperaturen $>1000{ }^{\circ} \mathrm{K}$ der Sauerstoff leicht im Kristall wandert.

Im übrigen sind bei Effekten, die in bezug auf die Leitfähigkeit von Oxyden lediglich mit einer Chemisorption von $\mathrm{O}_{2}$ verknüpft sind, im allgemeinen kleinere Wurzelexponenten als $6(<4)$ bzw. andere Beziehungen gefunden worden ( $\mathrm{H}_{\mathrm{AUFF}}, \mathrm{l}$. c. ${ }^{8}$ ), die überdies auch vom Sintergrad und von der Kristallkorngröße des polykristallinen Körpers abhängen dürften. Im Gegensatz hierzu verhalten sich aber z. $\mathrm{B} . \mathrm{BaO}$ und $(\mathrm{Ba}, \mathrm{Sr}) \mathrm{O}$, die sehr verschieden stark sintern und unterschiedliche Kristallkorngrößen besitzen, praktisch gleich.

In welcher Form nun - unter der Voraussetzung einer Sauerstoffaufnahme im Kristall - der Einbau von $O$ erfolgen kann und welcher Art die für die Defektleitung verantwortlichen Störstellen sind, läßt sich nicht entscheiden. Was den Einbau anbetrifft, so bleibt die Frage offen, ob der überschüssige Sauerstoff unter gleichzeitiger Bildung von Ba-Leerstellen (Ba-Ausbau und Bildung eines BaO-Moleküls an der Oberfläche) aufgenommen wird, oder ob infolge der mit der Temperatur steigenden Sснотткyschen Fehlordnung mit Kationen- und Anionenlücken bei $T>1000{ }^{\circ} \mathrm{K}$ im Kristall genügend Anionenleerstel-

20 C. Timmer, J. Appl. Phys. 28, 495 [1957]. 
len für die Besetzung durch $\mathrm{O}$ zur Verfügung stehen ${ }^{* * *}$ oder ob schließlich ein $\mathrm{O}$ gar unter Bildung eines Molekülions der Art $\mathrm{O}_{2}{ }^{2-}$, das nicht sehr viel mehr Raumbedarf als das einfache $\mathrm{O}^{2-}$-Ion hat, gleichsam im Zwischengitter aufgenommen wird.

Was die Beschaffenheit der die Defektleitung verursachenden Störstellen anbetrifft, so läßt sich zunächst nur sagen, daß eine Umladung des Kations bei Gegenwart überschüssigen Metalloids, wie sie z. B. beim $\mathrm{NiO}\left(\mathrm{Ni}^{2^{+}} \rightarrow \mathrm{Ni}^{3^{+}}\right)$angenommen wird, hier beim $\mathrm{BaO}$ der begrenzten Valenz des Kations wegen nicht möglich ist. Andererseits weist die Tatsache, daß bei anderen Metalloxyden mit Metallionen begrenzter Valenz (z. B. $\mathrm{ZnO}_{2} \mathrm{Al}_{2} \mathrm{O}_{3}$ usw.) in $\mathrm{O}_{2}$ keine Defektleitung, sondern höchstens eine Herabsetzung einer vorhandenen Überschußleitung beobachtet wird, darauf hin, daß in den Erdalkalioxyden Störstellen spezieller, zunächst noch unbekannter Art vorhanden sind, die möglicherweise mit der Fähigkeit dieser Oxyde zur Peroxydbildung in Beziehung stehen. Sсноттку ${ }^{4}$ hat diese Besonderheit der Peroxydhalbleiter in seiner Klassifikation der Störstellenhalbleiter (Gruppe der Superoxydhalbleiter) bereits 1939 berücksichtigt.

Unabhängig von einem speziellen Modell der Störstellen $\mathrm{im} \mathrm{BaO}$ ist auf Grund des $P_{\mathrm{O}_{2}}{ }^{1 / 6}$-Gesetzes zu schließen, daß bei hohen Temperaturen im Gebiet der reversiblen Sauerstoffabhängigkeit der Leitfähigkeit ein $\mathrm{O}$ zwei Defektelektronen zur Leitfähigkeit beisteuert und daher überwiegend zweiwertig negative Störstellen vorhanden sein sollten. In einer Diskussion über die Störstellen in Peroxydhalbleitern weist Sсноттку ${ }^{22}$ darauf hin, daß bei konstantem Druck mit zunehmender Temperatur infolge erhöhter Sauerstoffaufnahme auch mit verstärktem Auftreten von einwertigen Störstellen gerechnet werden darf, so daß das Verhältnis von zweiwertig negativen Störstellen zu einwertigen bei höheren Temperaturen kleiner sein könnte als bei tiefen Temperaturen. Das würde dann zur Folge haben, daß u. U. das $P_{\mathrm{O}_{2}}{ }^{1 / 6}$. Gesetz sich mit wachsender Temperatur zunehmend einem $P_{\mathrm{O}_{2}}{ }^{1 / 4}$-Gesetz nähern sollte. Diese nach Sсноттку mit steigender Temperatur auftretende systematische Abnahme von $x$ beim $P_{\mathrm{O}_{2}}{ }^{1 / x}$-Gesetz ist zwar bei den zusatzfreien Oxyden nicht (oder nicht

*** Unter Zugrundelegung ähnlicher Verhältnisse wie bei den polaren Alkalihalogeniden $\left(\mathrm{S}_{\text {EITZ }}{ }^{21}\right.$ ) ergäben sich für $\mathrm{BaO}$ bei Temperaturen $>1000{ }^{\circ} \mathrm{K}$ Sсноттку-Fehlstellen-Konzentrationen von $10^{17}$ bis $10^{18} / \mathrm{cm}^{3}$, die größenordnungsmäßig den für die beobachteten Leitwerte maßgeblichen Störstellendichten entsprechen dürften. deutlich) feststellbar, weil möglicherweise die Temperaturen bei den Messungen nicht hoch genug waren. Eine Abnahme von $x$ zeigt sich aber deutlich bei den Oxydproben mit den höherwertigen Zusatzionen $\mathrm{La}^{3^{+}}$oder $\mathrm{Y}^{3^{+}}$, die infolge ihrer Elektronenspenderwirkung bei gegebener Konzentration von überschüssigem Sauerstoff im Kristall die Zahl der von letzteren beigesteuerten Defektelektronen herabsetzen. Die überdies in diesem Falle beobachtete Abnahme von $x$ mit wachsender Temperatur ist möglicherweise auf den oben geschilderten Effekt zurückzuführen.

Daß schließlich in den alkalihaltigen Oxyden die Sauerstoffabhängigkeit der p-Leitfähigkeit - zunächst im Bereich höherer Drucke - praktisch aufgehoben wird, dürfte darauf zurückzuführen sein, daß die infolge des Einbaus der Alkaliionen im Gitter erzeugten Akzeptoren die Zahl der defektelektronenspendenden Störstellen bei der Sauerstoffaufnahme im Kristall weit übersteigt, so daß der Einfluß der Sauerstoffaufnahme in bezug auf die Leitfähigkeit nicht zur Wirkung kommen kann.

Das bei der Überschußleitung von $(\mathrm{Ba}, \mathrm{Sr}) \mathrm{O}$ in $\mathrm{H}_{2}$ beobachtete $P_{\mathrm{H}_{2}}{ }^{1 / 4}$-Gesetz entspricht - in ganz analoger Weise wie beim $\mathrm{O}_{2}$ - einer Lösung von $\mathrm{H}_{2}$ im Kristall gemäß

$$
\mathrm{H}_{2 \mathrm{~g}} \rightleftarrows 2 \mathrm{H}_{\mathrm{f}}^{+}+2 \ominus \text {. }
$$

Nach dem Massenwirkungsgesetz ergibt sich wieder:

$$
K \cdot P_{\mathrm{H} 2}=\left[\mathrm{H}_{\mathrm{f}}^{+}\right]^{2} \cdot[\Theta]^{2},
$$

und wegen der Proportionalität zwischen $\left[\mathrm{H}_{\mathrm{f}}^{+}\right],[\ominus]$ und $\sigma$ wird

$$
\sigma=K^{\prime} \cdot P_{\mathrm{H}_{2}}{ }^{1 / 4} .
$$

Die Art des Wasserstoffeinbaus ist entweder in Form von $\mathrm{H}^{+}$-Ionen auf $\mathrm{Z}$ wischengitterplätzen oder in Form von Donatoren als Hydroxylionen der Art $(\mathrm{OH} \cdot \odot)^{2^{-}}$ vorstellbar. Ein Einbau von $\mathrm{OH}^{-}$-Ionen mit einem Überschußelektron an Stelle von $\mathrm{O}^{2-}$-Ionen würde der Vorstellung von Lander und Thomas ${ }^{23}$ en: sprechen, die auf Grund der zuerst von Mollwo ${ }^{24}$ gefundenen Beeinflussung der Leitfähigkeit von (einkristallinem) $\mathrm{ZnO}$ durch $\mathrm{H}_{2}$ die Bildung von $(\mathrm{OH} \cdot \odot)^{2-}$-Störstellen annehmen. Überdies läge eine solche Annahme ganz im Sinne der neuerdings

21 F. Seitz, Rev. Mod. Phys. 26, 13 [1954].

22 Briefliche Mitteilung (1957).

23 J. J. Lander u. D. G. Thomas, J. Appl. Phys. 25, 1136 [1956].

24 E. Molnwo, Z. Phys. 138, 478 [1954]. 
von Plumlee ${ }^{16}$ auf Grund massenspektrographischer Untersuchungen an Oxydschichten und auf Grund thermochemischer Überlegungen entwickelten Vorstellung, daß mit solchen - auch für die Elektronenemission der Oxydkathode maßgeblichen - Donatoren in der Form $\left(\mathrm{OH}^{-} \cdot \ominus\right)^{2^{-}} \mathrm{zu}$ rechnen ist. Unsere Beobachtungen bezüglich des $\mathrm{H}_{2}$-Einflusses auf die Elektronenemission wären mit dieser Vorstellung
Plumlees, die indessen noch einer endgültigen $\mathrm{Be}$ stätigung bedarf, im Einklang.

Herrn Prof. Dr. W. Meyer und Herrn Prof. Dr. E. Krautz danke ich für die Ermöglichung der Durchführung dieser Arbeit in der OSRAM-Studiengesellschaft sowie für anregende Diskussionen. Zu besonderem Dank fühle ich mich Herrn Prof. Dr. W. Scнотткy für reges Interesse und fördernde Ratschläge sowie Herrn Prof. Dr. A. Schleede für viele Diskussionen verpflichtet.

\title{
Ultrarotabsorption von nÆleitendem Indiumarsenid*
}

\author{
Von Frank Matossi \\ Aus dem Institut für Elektrowerkstoffe, Universität Freiburg i. Br. \\ (Z. Naturforschg. 13 a, 767-770 [1958]; eingegangen am 2. Juni 1958)
}

\begin{abstract}
Die Absorption von n-leitendem InAs mit einem Netto-Donorgehalt von etwa $10^{16}$ per $\mathrm{cm}^{3}$ wurde nahe der Absorptionskante bei Temperaturen zwischen $120^{\circ} \mathrm{K}$ und $475{ }^{\circ} \mathrm{K}$ gemessen. Der Verlauf des Absorptionskoeffizienten kann durch zwei Exponentialfunktionen $\exp \left[h\left(v-v_{0}\right) / k T\right]$ beschrieben werden, wobei $T$ eine Effektivtemperatur von etwa $75^{\circ} \mathrm{K}$ oder $1800^{\circ} \mathrm{K}$ bedeutet. Diese Beobachtung wird durch Erweiterung einer von Dexter vorgeschlagenen Hypothese mittels des Verlaufs des Potentials im Grund- und angeregten Zustand als Funktion einer Konfigurationskoordinate gedeutet.
\end{abstract}

Die Ultrarotabsorption von InAs ist ebenso wie die anderer III - V-Verbindungen schon mehrmals untersucht worden ${ }^{1}$. Die vorliegende Untersuchung an n-leitendem Material legt besonderen Wert auf den Verlauf des Absorptionskoeffizienten im Gebiet scl:wacher Absorption, d.h. im langwelligen Ausläufer der Kantenabsorption und auf einen Vergleich mit vorliegenden Theorien oder Deutungsversuchen ${ }^{2}$.

Die Messungen wurden größtenteils mit einem Perkin-Elmer-Zweistrahl-Spektrograph Modell 21 mit $\mathrm{NaCl}$-Prisma vorgenommen. Nur zur genaueren Untersuchung des Absorptionsverlaufs bei stärkerer Absorption wurden Messungen mit dem EinstrahlModell 12 mit LiF-Prisma herangezogen. Das Untersuchungsmaterial hatte nach Ausweis seiner elektrischen Eigenschaften (Dr. J. Dixon) eine NettoDonorkonzentration von etwa $10^{16} \mathrm{~cm}^{-3}$. Dies eliminierte weitgehend Störungen durch überlagerte Freie-Träger-Absorption. Die Messungen wurden bei verschiedenen Temperaturen zwischen $120^{\circ} \mathrm{K}$

* Die Versuche zu dieser Arbeit wurden im U.S. Naval Ordnance Laboratory, Silver Spring, Maryland (Solid State Physics Division) durchgeführt. Ich danke der Leitung dieses Laboratoriums für die Erlaubnis, die Versuchsergebnisse nach meinem Weggang zu benutzen. Ebenso bin ich für wertvolle Hilfe bei der Präparation der InAs-Proben und für die Überlassung von Daten Herrn Dr. J. Dixon und Mrs. Dorothy Enright dankbar. und $475{ }^{\circ} \mathrm{K}$ in der gleichen Weise wie in einer Arbeit über p-InAs durchgeführt ${ }^{3}$.

\section{Ergebnisse}

Abb. 1 und 2 zeigen die Ergebnisse in halblogarithmischer Darstellung. Es standen Messungen an Proben verschiedener Dicke (zwischen 70 und $500 \mu$ ) zur Verfügung, die allerdings wegen verschiedener Qualität der Politur etwas verschiedene Absolutwerte des Absorptionskoeffizienten $\alpha$ ergaben, während die Neigungen der Kurven unabhängig von der Dicke waren. Als zuverlässigste Messungen wurden die angesehen, die die niedrigsten $\alpha$-Werte ergaben. Die Proben waren polykristallin, jedoch im allgemeinen mit nur wenigen Kristalliten im durchstrahlten Querschnitt. Als Reflexionsverlust wurde auf Grund eigener Messungen unabhängig von der Wellenlänge $50 \%$ angenommen. Auch hier ist natürlich der Absolutwert unsicher, und zwar um etwa $2 \%$.

1 Vgl. z. B. den Bericht von H. Welker und H. Weiss in „Advances in Solid State Physics", hgg. von F. Seitz und D. Turnbull, 3, 1 [1956].

2 D. L. Dexter in "Photoconductivity Conference“, hgg. von R. G. Breckenridge u. a., John Wiley \& Sons, New York 1956, p. 155.

3 F. Matosi u. F. Stern, Phys. Rev. 111, Nr. 2 [1958]. 